\title{
Funny business in branching space-times: infinite modal correlations
}

\author{
Thomas Müller • Nuel Belnap • Kohei Kishida
}

Received: 26 May 2006 / Accepted: 4 July 2007 / Published online: 10 August 2007

(C) Springer Science+Business Media B.V. 2007

\begin{abstract}
The theory of branching space-times is designed as a rigorous framework for modelling indeterminism in a relativistically sound way. In that framework there is room for "funny business", i.e., modal correlations such as occur through quantummechanical entanglement. This paper extends previous work by Belnap on notions of "funny business". We provide two generalized definitions of "funny business". Combinatorial funny business can be characterized as "absence of prima facie consistent scenarios", while explanatory funny business characterizes situations in which no localized explanation of inconsistency can be given. These two definitions of funny business are proved to be equivalent, and we provide an example that shows them to be strictly more general than the previously available definitions of "funny business".
\end{abstract}

Keywords Correlations $\cdot$ Modality $\cdot$ Indeterminism

\section{Introduction}

We should take indeterminism seriously as an objective feature of our world. This means that there are events whose outcomes are not determined before they occur. Given relativity theory, such events must be localized in bounded space-time regions or, to use an idealization, at space-time points. The theory of branching space-times (Belnap 1992) was designed to provide a formally rigorous framework for the analysis of questions pertaining to objective indeterminism. We will denote that theory,

T. Müller $(\varangle)$

Institut für Philosophie, Lennéstr. 39, Universität Bonn, 53113 Bonn, Germany

e-mail: Thomas.Mueller@uni-bonn.de

N. Belnap · K. Kishida

Department of Philosophy, University of Pittsburgh, 1001 Cathedral of Learning,

Pittsburgh, PA 15260, USA 
which will be employed in this paper, as "BST-92" in order to distinguish it from other approaches to "branching space-times" in the literature (cf. Belnap 2003 for an overview; BST-92 is outlined in the Appendix).

If indeterminism is bound to localized events, it makes sense to ask in which way such events may combine. We know from probability theory that in combining probability spaces, correlations may show up. A probabilistic correlation is signalled by the fact that the probability of a combined event is not equal to the product of the probabilities of the subevents. We will not be concerned with probabilities here, but with a question that is conceptually prior: Given that some outcomes of localized events are individually possible, we wish to understand which combinations of such outcomes are possible. Thus, our topic may be called "modal correlations".

If anything, modal correlations cry out for explanation even more than probabilistic correlations. Consider two coins tossed independently at different locations. It would certainly be weird if the probabilities for the four possible combined outcomes were not equal to the respective products of the individual probabilities-e.g., if both coins were fair (probability of "heads" equal to 0.5 ), but the probabilities of "heads-heads" was, say, 0.1 instead of the expected 0.25 . Still, that probabilistic weirdness would be in accord with ordinary expectations about the modal structure of the combined set-up: given two independent set-ups with two possible outcomes each, there would still be four possible combined outcomes. It would be much weirder still if not just the probabilistic, but the underlying modal structure of the combined set-up showed correlations-e.g., if the "head-head" outcome was impossible, even though for each coin, the "head" outcome was possible. Yet, nature seems to exhibit exactly such modal correlations in certain quantum-mechanical set-ups. ${ }^{1}$ What is going on in these cases? Certainly this is "funny business", and we will use this expression to signal intuitively worrisome modal correlations.

The notion of modal correlations, or funny business, has been analyzed by Belnap in two successive papers (Belnap 2002, 2003). The upshot of these papers was that there seems to be a stable notion of funny business in BST-92, which can be characterized in four equivalent ways, each of which casts some additional light on notions of modal correlations, causation, and screening-off. In this paper, we push the analysis one step further by tackling infinite cases. We will give two new, equivalent definitions of funny business in BST-92 that properly generalize the existing notions. These two definitions will be given in the language of transitions, which concept has been used in a BST-92 based analysis of causation (Belnap 2005). Even though our paper becomes technical in places, the focus is on understanding the conceptual structure of modal correlations.

\footnotetext{
1 Well-known cases are the EPR (Einstein et al. 1935) set-up and, more prominently, the GHZ (Greenberger et al. 1989) set-up; cf. Bub (1997) for an overview. It may be argued that due to experimental uncertainties, the perfect (anti-)correlations presupposed in these idealized set-ups can never be achieved, so that what is at issue is never modal, but always probabilistic correlation. We hold that conclusion to be premature. Certainly, for conceptual reasons one should understand modal correlations first. Investigations into probabilistic BST-92 (Müller 2005) underline this attitude: It seems that in order to do probability theory at all, one has to presuppose that no modal correlations are present. If so, then one had better understand the absence of what one is presupposing.
} 
The structure of our paper is as follows: We first introduce our notation and some key definitions of BST-92 as well as the four existing notions of funny business (Sect. 2). Then we provide three BST-92 models as test cases (Sect. 3). These models will be used to motivate our new definitions, given in Sect. 4 and 5. Appendix A contains some formal details of BST-92.

\section{Notation and proposed definitions of funny business}

The label "BST-92" stands for the theory of branching space-times as laid out in Belnap (1992). We define some of the key notions of that theory in Appendix A. The interested reader is referred to Belnap $(2002,2003)$, where additional motivation is given.

BST-92 describes alternative courses of events in terms of families of histories branching at space-time points. These histories are carved from an underlying partial ordering $\langle W, \leq\rangle$. In the following, $h$ stands for a history (a maximally detailed possible course of events, corresponding, e.g., to a single Minkowski space-time), $I$ for an initial event (occurring in at least one, but commonly in many histories), and $\mathbf{O}$ for an outcome event, starting to occur in at least one history. An outcome event consists of one or more outcome chains $O \in \mathbf{O}$. The set of histories $h$ for which $h \cap \mathbf{O} \neq \emptyset$ is denoted $H_{\langle\mathbf{O}\rangle}$. Different histories split off at one or more points, we write " $h_{1} \perp_{e} h_{2}$ " for "history $h_{1}$ splits off from history $h_{2}$ at $e$." Such splitting introduces a partition $\Pi_{e}$ of the set $H_{(e)}$ of histories containing $e$. More generally, $\Pi_{I}$ is the corresponding partition of the set $H_{[I]}$ of histories containing the initial $I$, and $\Pi_{I}\langle h\rangle$ is that member of that partition containing $h$. Two initials can be space-like related, $I_{1}$ SLR $I_{2}$, meaning that none of their members stand in the relation of causal precedence. A basic transition is a pair $\langle e, H\rangle$, written $e \longmapsto H$, where $H \in \Pi_{e}$. Basic transitions are the fundamental indeterministic structures within branching space-times.

"Funny business" intuitively means that there is a spatio-temporal/modal structure that has some special (funny) properties that strike one as odd, weird, or counterintuitive. (Still, for all we know, such structures exist; witness the large literature on quantum-mechanical correlations and EPR-Bell-type cases; cf. note 1 for some references.) Belnap $(2002,2003)$ proposed four notions of "funny business" and showed them to be equivalent. We refer the reader to these papers for details. The following definitions list the four notions.

Definition 1 (Primary SLR modal-correlation funny business) Two initials $I_{1}$ and $I_{2}$ together with two outcome-determining histories $h_{1}$ and $h_{2}\left(I_{i} \subseteq h_{i}\right.$ for $\left.i=1,2\right)$ constitute a case of primary SLR modal-correlation funny business iff $I_{1}$ SLR $I_{2}$, but $\Pi_{I_{1}}\left\langle h_{1}\right\rangle \cap \Pi_{I_{2}}\left\langle h_{2}\right\rangle=\emptyset$.

Thus, two intuitively independent (since SLR ) initials and outcomes constitute a case of funny business if the outcomes do not combine smoothly. This definition will be generalized to what we call combinatorial funny business below.

Definition 2 (Some-cause-like-locus-not-in-past funny business) An initial I, a scattered outcome event $\mathbf{O}$ and a history $h$ constitute a case of some-cause-like-locus-notin-past funny business iff $I$ is a cause-like locus for $\mathbf{O}$ w.r.t. $h$, but no member of $I$ lies in the causal past of any member of $\mathbf{O}$. 
According to this definition, funny business is linked to something like "spooky action at a distance": There is a cause-like initial $I$ for $\mathbf{O}$, but that initial does not act in the causal past of $\mathbf{O}$.

Definition 3 (No-prior-screener-off funny business) A pair of scattered outcome events $\mathbf{O}_{1}$ and $\mathbf{O}_{2}$ constitute a case of no-prior-screener-off funny business iff

1. Each of $\mathbf{O}_{1}$ and $\mathbf{O}_{2}$ is individually consistent, i.e., $H_{\left\langle\mathbf{O}_{1}\right\rangle} \neq \emptyset$ and $H_{\left\langle\mathbf{O}_{2}\right\rangle} \neq \varnothing$,

2. $H_{\left\langle\mathbf{O}_{1}\right\rangle}$ is inconsistent with $H_{\left\langle\mathbf{O}_{2}\right\rangle}$, i.e., $H_{\left\langle\mathbf{O}_{1}\right\rangle} \cap H_{\left\langle\mathbf{O}_{2}\right\rangle}=\emptyset$, but

3. $\neg \exists e \exists O_{1} \in \mathbf{O}_{1} \exists O_{2} \in \mathbf{O}_{2}\left[e<O_{1}\right.$ and $e<O_{2}$ and $\forall h \in H_{(e)}\left[\left(\Pi_{e}\langle h\rangle \cap H_{\left\langle\mathbf{O}_{1}\right\rangle}=\right.\right.$ $\emptyset)$ or $\left.\left(\Pi_{e}\langle h\rangle \cap H_{\left\langle\mathbf{O}_{1}\right\rangle}=\emptyset\right)\right]$.

In such a case, two outcomes are individually consistent yet inconsistent, and we are lacking an intuitively satisfactory explanation of the inconsistency, which would have to be in terms of a prior event $e$ separating off the two inconsistent outcomes. This notion will be generalized to what we call explanatory funny business below.

Definition 4 (No-prior-common-cause-like-locus funny business) A pair of scattered outcome events $\mathbf{O}_{1}$ and $\mathbf{O}_{2}$ together with a pair of histories $h_{1}$ and $h_{2}$ constitute a case of no-prior-common-cause-like-locus funny business iff

1. Each of $\mathbf{O}_{1}$ and $\mathbf{O}_{2}$ is individually consistent, as witnessed by $h_{1}$ and $h_{2}$ (i.e., $h_{1} \in H_{\left\langle\mathbf{O}_{1}\right\rangle}$ and $\left.h_{2} \in H_{\left\langle\mathbf{O}_{2}\right\rangle}\right)$,

2. $H_{\left\langle\mathbf{O}_{1}\right\rangle}$ is inconsistent with $H_{\left\langle\mathbf{O}_{2}\right\rangle}$, i.e., $H_{\left\langle\mathbf{O}_{1}\right\rangle} \cap H_{\left\langle\mathbf{O}_{2}\right\rangle}=\emptyset$, but

3. $\neg \exists e \exists O_{1} \in \mathbf{O}_{1} \exists O_{2} \in \mathbf{O}_{2}\left[e<O_{1}\right.$ and $e<O_{2}$ and $\left.h_{1} \perp_{e} h_{2}\right]$.

This definition gives a variant of the idea behind the previous definition.

The main result of Belnap (2003) is to establish the equivalence of these four notions. Thus, we have the following Theorem:

Theorem 1 In a BST-92 model $\langle W, \leq\rangle$, there is a case of primary SLR modal-correlation funny business iff there is a case of some-cause-like-locus-not-in-past funny business iff there is a case of no-prior-screener-off funny business iff there is a case of no-prior-common-cause-like-locus funny business.

In this paper, we propose to extend the previous analysis of "funny business" in such a way as to cover certain infinite structures that are intuitively "funny", but which are not covered by the existing definitions. Also, in line with the project of explaining causation in terms of basic transitions, initiated by Belnap (2005), the new definition will apply to sets of transitions, which may be easier to handle than the spatio-tempo$\mathrm{ral} /$ modal structures involved in the previous definitions.

\section{Test cases}

In this section we will construct three BST-92 structures. One is clearly innocent as regards funny business, while the other two are intuitively "funny", since they both exhibit space-like correlations. ${ }^{2}$ For all structures, we will proceed in two steps: We

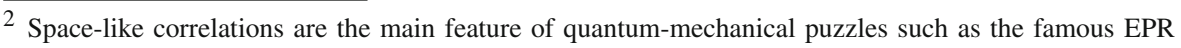
argument. Cf. note 1 for some references.
} 


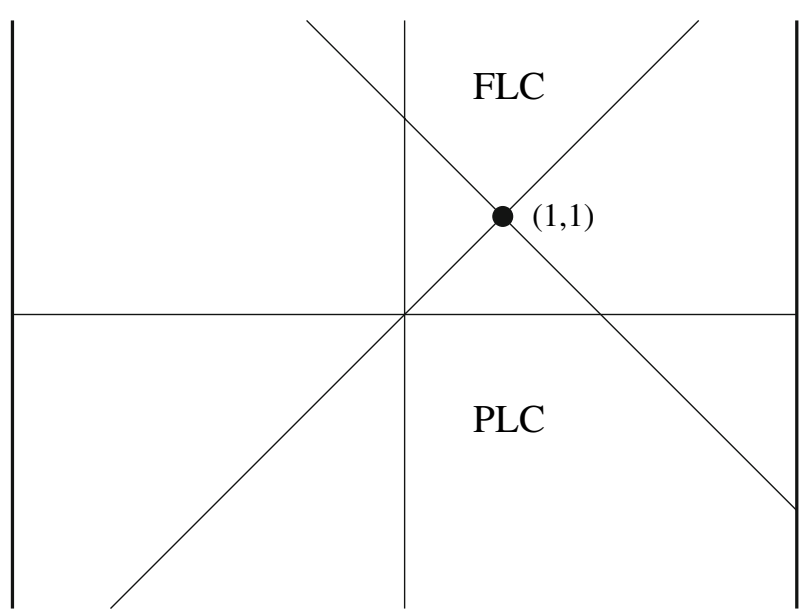

Fig. $1 \mathcal{M}$, the two-dimensional Minkowski plane. The figure shows the past light cone $(P L C)$ and future light cone $(F L C)$ for the point $(1,1)$. For all $(x, y) \in P L C$, we have $(x, y)<_{M}(1,1)$, while for all $(x, y) \in F L C,(1,1)<_{M}(x, y)$

will first define the structures and prove that they fulfill the postulates of BST-92. In a second step, we will give an intuitive assessment of whether the structures exhibit "funny business", and check whether the existing definitions of funny business apply in the appropriate way. For the last structure, $M_{2}$, this will not be so, and this fact will then motivate our extended definitions of funny business to be presented below.

Our first two structures will be derived from a 2-dimensional Minkowski space-time $\mathcal{M}$ (cf. Fig. 1). This is the structure

$$
\mathcal{M}=\left\langle M,<_{M}\right\rangle
$$

where the base set $M=\mathbb{R}^{2}$ is the set of pairs of real numbers (the Euclidean plane), and the (causal) partial ordering $<_{M}$ is defined within light cones (taking the speed of light $c=1$ ), as follows:

$$
(x, y)<_{M}\left(x^{\prime}, y^{\prime}\right) \quad \text { iff } \quad\left|x-x^{\prime}\right| \leq\left|y-y^{\prime}\right| \text { and } y<y^{\prime} .
$$

Furthermore, our structures will exhibit indeterminism in the form of a 2-way splitting, with the outcomes denoted as " 0 " and " 1 ". You may think of these indeterministic events as coin tosses, or as measurements of the spin projection of a spin-1/2 particle along some axis, or of some other simple indeterministic event. We will not look at probabilistic setups (which would require an extension of the basic structure of BST92; cf. Weiner and Belnap (2006) and Müller (2005)), so by "correlations" we mean modal correlations, or perfect correlations.

As a warm-up, and in order to introduce some techniques that we will use in the "funny" models, we first present a straightforward BST-92 structure without funny business, which you may think of as modelling two coins tossed independently at different locations. 
3.1 $M_{0}$ : no funny business

We start with the set

$$
C=d f\{(-1,0),(1,0)\},
$$

which will be our two choice points (the space-time locations at which the coin tosses happen; at these points, the outcomes are not yet fixed). We will use $p, p^{\prime}$, etc., to range over $M$, and we employ the notation

$$
J^{-}(p){ }_{d f}\left\{p^{\prime} \in M \mid p^{\prime}<_{M} p\right\}
$$

to denote the causal past (the past light cone) of $p$ (excluding the point $p$ itself).

Our BST-92 structure $M_{0}$ is based on the set $W$, defined as

$$
W={ }_{d f}\left\{\langle p, G\rangle \mid p \in M, G \subseteq C \cap J^{-}(p)\right\} .
$$

On $W$ we define a partial ordering < via

$$
\left\langle p_{1}, G_{1}\right\rangle<\left\langle p_{2}, G_{2}\right\rangle \quad \text { iff } \quad p_{1}<_{M} p_{2} \quad \text { and } \quad G_{1}=G_{2} \cap J^{-}\left(p_{1}\right) .
$$

The interpretation of the set and its ordering is as follows: A point $e=\langle p, G\rangle \in W$ stands for the space-time location $p \in M$, considered in a history in which exactly the initials from $G$, which lie in the past of $p$, show outcome " 0 ", while all other initials that are in the past of $p$ show outcome " 1 ". (Thus, the elements of $W$ contain no information about the outcomes of choice points that are space-like related to, or in the causal future of, $p$.) The ordering then says that two elements of $W$ stand in the ordering relation exactly if their spatio-temporal locations are ordered appropriately and the information on " 0 " and " 1 " outcomes from the lower point is consistent with that from the higher point. (If you wish, you may read this as some kind of "no backward branching" requirement.)

We now show that $M_{0}=\langle W,<\rangle$ fulfills the postulates of BST-92.

Lemma 1 (Histories in $M_{0}$ )

The histories in $M_{0}$ are exactly the sets

$$
h_{i}=\left\{\langle p, G\rangle \mid p \in M, G=C_{i} \cap J^{-}(p)\right\}, \quad i=1, \ldots, 4,
$$

where the $C_{i}$ are the four subsets of $C: \emptyset,\{(-1,0)\},\{(1,0)\}$, and $C$ itself.

Proof " $\Leftarrow$ ": Let $h_{i}$ be one of the mentioned sets. We need to show that that is a history in $M_{0}$, i.e., that it is a maximally directed set. For directedness, take $e_{1}=\left\langle p_{1}, C_{i} \cap J^{-}\left(p_{1}\right)\right\rangle, e_{2}=\left\langle p_{2}, C_{i} \cap J^{-}\left(p_{2}\right)\right\rangle \in W$. As $p_{1}$ and $p_{2}$ have an upper bound, $p$, in $M$, the point $\left\langle p, C_{i} \cap J^{-}(p)\right\rangle \in W$ is an upper bound for $e_{1}$ and $e_{2}$ in $W$. For maximality, assume that $e=\langle p, G\rangle \notin h_{i}$ and note that $e^{\prime}=\left\langle p, C_{i} \cap J^{-}(p)\right\rangle \in h_{i}$. If $h_{i} \cup\{e\}$ were a subset of some directed set $h^{*}$, then $h^{*}$ would have to contain an 
upper bound $e^{*}=\left\langle p^{*}, G^{*}\right\rangle$ above both $e$ and $e^{\prime}$. But then by the definition of the ordering, we would have to have $G=G^{*} \cap J^{-}(p)=C_{i} \cap J^{-}(p)$, contradicting $e \notin h_{i}$.

" $\Rightarrow$ ": Let $h$ be a maximally directed subset of $W$. We need to show that $h=h_{i}$ for one of the $h_{i}$ defined above.

Assume for reductio that $h$ does not contain a point $e_{1}=\left\langle p_{1}, G_{1}\right\rangle$ such that $(-1,0)<_{M} p_{1}$. Let $e=\langle p, G\rangle \in h$. Since $(-1,0) \notin J^{-}(p)$ by assumption, and so is not in $G \subseteq C \cap J^{-}(p)$, either $G=\emptyset$ or $G=\{(1,0)\}$. Hence either $G=C_{1} \cap J^{-}(p)$ or $G=C_{3} \cap J^{-}(p)$, so that $e \in\left(h_{1} \cup h_{3}\right)$, and accordingly $h \subseteq\left(h_{1} \cup h_{3}\right)$. Therefore either $h=h_{1}$ or $h=h_{3}$ (as proven in Belnap (1992)); but then the definition of the $h_{i}$ contradicts the assumption, so that $h$ must after all contain a point $e_{1}=\left\langle p_{1}, G_{1}\right\rangle$ such that $(-1,0)<_{M} p_{1}$. An exactly similar argument establishes that $h$ contains a point $e_{2}=\left\langle p_{2}, G_{2}\right\rangle$ such that $(1,0)<_{M} p_{2}$.

Given that $h$ contains both such an $e_{1}$ and such an $e_{2}$, since $h$ is directed, it must contain an upper bound $e=\langle p, G\rangle$ for $e_{1}$ and $e_{2}$, so $(-1,0)<_{M} p,(1,0)<_{M} p$. By the definition of $W$, we have $G \subseteq C$, so $G=C_{i}$ for one of $i=1, \ldots, 4$. We can now show that $h \subseteq h_{i}$. Otherwise, let $e^{\prime} \in h-h_{i}$, i.e, $e^{\prime}=\left\langle p^{\prime}, G^{\prime}\right\rangle$ with $G^{\prime} \neq C_{i} \cap J^{-}\left(p^{\prime}\right)$. By directedness of $h$, there must be $e^{*}=\left\langle p^{*}, G^{*}\right\rangle \in h$ above both $e$ and $e^{\prime}$. Now from $e<e^{*}$ we have $G=C_{i}=G^{*} \cap J^{-}(p)=G^{*}$, but then by $e^{\prime}<e^{*}$ we also have $G^{\prime}=G^{*} \cap J^{-}\left(p^{\prime}\right)=C_{i} \cap J^{-}\left(p^{\prime}\right)$, showing that $e^{\prime} \in h_{i}$, contrary to assumption. Finally, in virtue of the maximality of $h, h \subseteq h_{i}$ implies that $h=h_{i}$.

In order to establish the other postulates of BST-92, note that density and the existence of suprema and infima carry over from $\mathcal{M}$. It remains to establish the prior choice postulate.

Lemma 2 ( $M_{0}$ satisfies the prior choice postulate) $M_{0}$ satisfies the prior choice postulate, i.e., if $O$ is a lower bounded chain in $h_{i}-h_{j}$, there is a point $e \in h_{i} \cap h_{j}$ such that e is a lower bound for $O$ and e is maximal in $h_{i} \cap h_{j}$.

Proof Let $O \subset h_{i}-h_{j}$ be a lower bounded chain. Set

$$
\Delta_{i j}=\left(C_{i}-C_{j}\right) \cup\left(C_{j}-C_{i}\right)
$$

the set of indeterministic initials whose outcomes distinguish $h_{i}$ from $h_{j}$.

(1) If $p_{k} \in \Delta_{i j}$, then $\left\langle p_{k}, \varnothing\right\rangle$ is maximal in $h_{i} \cap h_{j}$ : Take $p_{k} \in \Delta_{i j}$. By Lemma 1 , $\left\langle p_{k}, \varnothing\right\rangle \in h_{i} \cap h_{j}$. For maximality, let $e=\langle p, G\rangle \in h_{i}$ s.t. $\left\langle p_{k}, \varnothing\right\rangle<e$. Then $G=C_{i} \cap J^{-}(p)$ (as $e \in h_{i}$ ), but as $p_{k}<_{M} p$ and $p_{k} \in \Delta_{i j}$, it must be that $G \neq C_{j} \cap J^{-}(p)$, so that $e \notin h_{j}$.

(2) For $e=\langle p, G\rangle \in O$, from $e \in h_{i}-h_{j}$ we get $G=C_{i} \cap J^{-}(p) \neq C_{j} \cap J^{-}(p)$, so there is a $p_{k} \in \Delta_{i j}$ s.t. $p_{k}<_{M} p$. It remains to prove that there is $p_{k} \in \Delta_{i j}$ s.t. for all $e=\langle p, G\rangle \in O$ we have $p_{k}<_{M} p$, since then $\left\langle p_{k}, \emptyset\right\rangle<O$, and maximality was established above. So assume for reductio that there is no such $p_{k}$, i.e., for all $p_{k} \in \Delta_{i j}$ there is $e_{k} \in O$ s.t. $\left\langle p_{k}, \emptyset\right\rangle \nless e_{k}$. Let $E$ contain such a witness $e_{k}$ for each $p_{k} \in \Delta_{i j}$ : we set $E={ }_{d f}\left\{e_{k} \mid p_{k} \in \Delta_{i j}\right\}$. Now $E \subseteq O$, so $E$ is a chain in $h_{i}-h_{j}$, and as $\Delta_{i j}$ is finite, $E$ contains a least element, $e_{0}=\left\langle p_{0}, G_{0}\right\rangle$. Now we cannot have $p_{k}<p_{0}$ for any $p_{k} \in \Delta_{i j}$, for else by transitivity (noting that $E$ 
is a chain) we would have $\left\langle p_{k}, \emptyset\right\rangle<e_{0} \leq e_{k}$, contrary to the construction of $E$. That is, $\Delta_{i j} \cap J^{-}\left(p_{0}\right)=\emptyset$. But then $G_{0}=C_{i} \cap J^{-}\left(p_{0}\right)=C_{j} \cap J^{-}\left(p_{0}\right)$, so that $e_{0} \in h_{j}$, contradicting $e_{0} \in O$.

The structure $M_{0}$ does not exhibit any strange correlations- to each combination of outcomes of the indeterministic events in $M_{0}$ there corresponds a history. As we would expect, $M_{0}$ is not a case of funny business in the technical sense either.

Fact 1 The structure $M_{0}$ does not exhibit primary space-like related modal-correlation funny business.

Proof The only candidate for primary space-like related modal-correlation funny business are the initials $I_{1}=\{\langle(-1,0), \varnothing\rangle\}$ and $I_{2}=\{\langle(1,0), \varnothing\rangle\}$. However, any combination of outcomes is consistent, as witnessed by the four histories $h_{1}, \ldots, h_{4}$.

\section{2 $M_{1}$ : EPR-like funny business}

For the model $M_{1}$ we start with the set $C$ as above. However, there will be a new intermediate step: We select a proper subset $\mathcal{C}$ of the powerset of $C$ to mark the possible combinations of outcomes, rather than selecting the full powerset as above. Thus, we set $\mathcal{C}=\{\emptyset,\{(-1,0)\},\{(1,0)\}\}$. The definition of $W$ is almost as above:

$$
W={ }_{d f}\left\{\left\langle p, G_{i}\right\rangle \mid p \in M, G_{i}=C_{i} \cap J^{-}(p), C_{i} \in \mathcal{C}, i=1, \ldots, 3\right\}
$$

The partial ordering is defined exactly as above. In order to show that $M_{1}=\langle W,<\rangle$ fulfills the postulates of BST-92, we follow the proof for $M_{0}$ almost to the letter. The history lemma reads:

Lemma 3 (Histories in $M_{1}$ ) The histories in $M_{1}$ are exactly the sets

$$
h_{i}=\left\{\left\langle p, G_{i}\right\rangle \mid p \in M, G_{i}=C_{i} \cap J^{-}(p)\right\}, \quad i=1, \ldots, 3,
$$

where the $C_{i}$ are the elements of $\mathcal{C}$.

The proof of the Lemma is as above, and the prior choice principle is also established exactly as already shown. (It helps to note that $M_{1}$ is a substructure of $M_{0}$, with exactly the points $\langle p,\{(-1,0),(1,0)\}\rangle$ missing from $W$.)

The structure $M_{1}$ is weird: While the two choice points are space-like related, and each could have outcome " 0 " (as witnessed by $h_{2}$ and $h_{3}$ ), there is no history in which they both have the outcome " 0 ". This is exactly the type of set-up for which the term "funny business" was coined, and accordingly, the definition applies:

Fact 2 The structure $M_{1}$ exhibits primary SLR modal-correlation funny business.

Proof Consider the initials $I_{1}=\{\langle(-1,0), \varnothing\rangle\}$ and $I_{2}=\{\langle(1,0), \varnothing\rangle\}$. They are spacelike related, and histories $h_{2}$ and $h_{3}$ witness that each can have outcome " 0 ". However, there is no history in $M_{1}$ that witnesses the combined outcome. 


\section{3 $M_{2}$ : infinite funny business}

In order to show that there can be funny business that is not a case of the existing definitions, we use the following combinatorics: Let there be denumerably many SLR choice points (labelled by $n \in \mathbb{N}$ ), each with binary splitting (outcomes 0 and 1 ). We will construct a model in which the histories correspond to exactly those combinations of outcomes in which only finitely many 0's occur. This will result in intuitively "funny combinatorics", as each choice point can have outcome 0 , but the combination "all outcomes are 0" cannot occur. However, there will be no case of primary SLR modal-correlation funny business (Def. 1). To see this, let $I_{1}$ and $I_{2}$ be subsets of $\mathbb{N}$ s.t. $I_{1} \cap I_{2}=\emptyset$; this exhausts all possible choices of SLR initials. Now let histories $h_{1}$ and $h_{2}$ witness some outcome of $I_{1}$ and of $I_{2}$, respectively. As histories correspond to exactly those combinations of outcomes with finitely many 0's, the designated combined outcome for the initial $I_{1} \cup I_{2}$ involves only finitely many 0 's as well, and thus there is a history $h^{*}$ witnessing the combined outcome. Thus there is no case of primary SLR modal-correlation funny business-but there is obviously funny business going on!

An attempt to construct a model with these combinatorics along the lines of the construction of $M_{1}$ results in an instructive failure. Using Minkowski space-time as the background, we specify the points $(0, n), n \in \mathbb{N}$, as designated choice points:

$$
C=\{(n, 0) \mid n \in \mathbb{N}\} .
$$

The set of allowed combinations, $\mathcal{C}$, is the set of finite subsets of $C$ :

$$
\mathcal{C}=\left\{C_{i} \subset C \mid C_{i} \text { is finite }\right\}
$$

The construction of $W$ and of the ordering then follows the lines of the construction of $M_{1}$ exactly. As in the proof of Lemma 1 , one sees that for each $C_{i} \in \mathcal{C}$, the set

$$
h_{i}=\left\{\langle p, G\rangle \mid p \in M, G=C_{i} \cap J^{-}(p)\right\}
$$

is indeed a history. However, there are histories that are not of this form. To see this, consider the "vertical" chain $\left\langle p_{n}, G_{n}\right\rangle$ of points with $p_{n}=(0, n)$ and $G_{n}=$ $C \cap J^{-}\left(p_{n}\right), n \in \mathbb{N}$. Each $G_{n}$ is finite, so the points $\left\langle p_{n}, G_{n}\right\rangle$ do belong to the envisaged structure, and by the construction of the ordering,

$$
\left\langle p_{n}, G_{n}\right\rangle \leq\left\langle p_{n+1}, G_{n+1}\right\rangle .
$$

Thus, we have a chain (thus, also a directed set), and by Zorn's Lemma, there is a history $h^{*}$ (a maximal directed set) containing the chain. Now in $h^{*}$, all choice points have the 0 outcome, thus thwarting the attempt to implement the "funny" combinatorics in analogy with $M_{1}{ }^{3}$

\footnotetext{
${ }^{3}$ More elaborate attempts, such as choosing $C$ to be the full real line and $\mathcal{C}$ the set of null sets, may give the right histories, but usually lead to a failure of the prior choice principle.
} 
The following construction, which is much sparser, does provide a means for implementing the sought-for combinatorics. Roughly, one takes denumerably many copies of the real line, binds them together at some point "far back", and only combines those lines by the ordering that reflect the sought-for combinatorics. You may think of the position along the real line as an analogue of temporal location, while the other aspects of the construction give rise to space-like separation. Thus, let $\mathbb{F}$ be the set of all functions $f: \mathbb{N} \rightarrow\{0,1\}$ such that for only finitely many $n \in \mathbb{N}, f(n)=0$.

Now set $W=W_{0} \cup W_{1} \cup W_{2} \cup W_{3}$ to be the union of the following four Cartesian products:

$$
\begin{aligned}
& W_{0}=(-\infty, 0], \\
& W_{1}=(0,1] \times \mathbb{N}, \\
& W_{2}=(1,2) \times \mathbb{N} \times\{0,1\}, \\
& W_{3}=[2, \infty) \times \mathbb{F} .
\end{aligned}
$$

For each tuple $e \in W_{i}$, its first coordinate pictures "temporal location" along the real line, while the other coordinates picture the "space-like position" in the bundle $W$. Our ordering, $<$, is the transitive closure of the following relations (a) within the $W_{i}$ and (b) between elements of $W_{i}$ and $W_{i+1}$ :

(a) For $e, e^{\prime}$ from the same $W_{i}: e<e^{\prime}$ iff the first coordinate of $e$ is smaller than that of $e^{\prime}$ and the other coordinates are the same. E.g., for $x, y \in W_{0}$, let $x<y$ in $W$ iff $x<y$ as reals; and for $(x, n),(y, m) \in W_{1}$, let $(x, n)<(y, m)$ iff $x<y$ and $n=m$.

(b.0) $x<(y, n)$ for every $x \in W_{0}$ and $(y, n) \in W_{1}$.

(b.1) For $(x, n) \in W_{1}$ and $(y, m, i) \in W_{2}:(x, n)<(y, m, i)$ iff $n=m$.

(b.2) For $(x, n, i) \in W_{2}$ and $(y, f) \in W_{3}:(x, n, i)<(y, f)$ iff $f(n)=i$.

It is clear that $(x, a)<(y, b)$ only if $x<y$, and hence $<$ is a (strict) partial ordering. Figure 2 illustrates this ordering and indicates where the boundary points belong.

In order to prove that this structure fulfills the postulates of BST-92, we first note that the histories are in one-to-one correspondence with the members of $\mathbb{F}$.

Lemma 4 The histories in $M_{2}$ are exactly the sets

$$
h_{f}={ }_{d f} W_{0} \cup W_{1} \cup\left\{(x, n, i) \in W_{2} \mid f(n)=i\right\} \cup\left\{(x, g) \in W_{3} \mid g=f\right\}
$$

with $f \in \mathbb{F}$.

Proof " $\Leftarrow$ ": For $f \in \mathbb{F}, h_{f}$ is maximally directed. Directedness is established easily (use some $(y, f)$ with $y$ large enough). Maximality also follows straightforwardly from the definition of the ordering, noting that as $W_{0} \cup W_{1} \subset h_{f}$, an element $e \in W-h_{f}$ must be of the form $\left(x, n, i^{\prime}\right)$ with $f(n) \neq i^{\prime}$, or $\left(x, f^{\prime}\right)$ with $f \neq f^{\prime}$.

" $\Rightarrow$ ": In the other direction, let $D$ be a nonempty directed subset of $W$. We will show that there is $f \in \mathbb{F}$ for which $D \subseteq h_{f}$. By maximality of the $h_{f}$, we will thereby have established our claim. We distinguish three cases: 


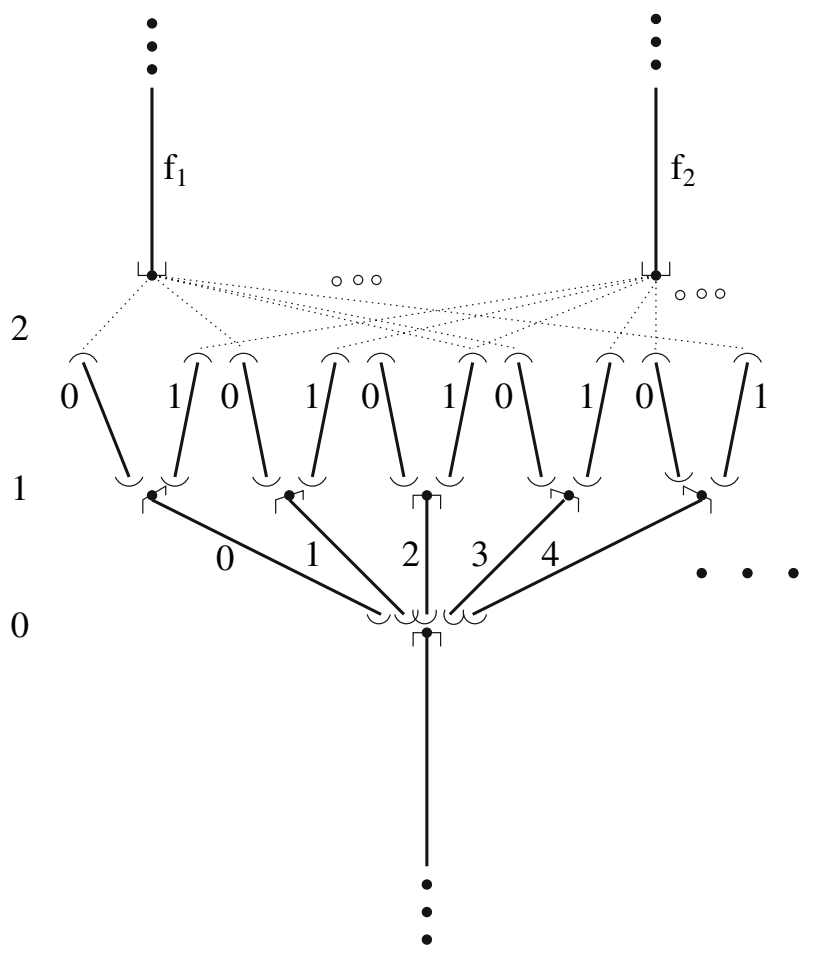

Fig. 2 The ordering relations in the model $M_{2}$

(i) If $D \subset W_{0} \cup W_{1}$, we have $D \subset h_{f}$ for any $f \in \mathbb{F}$, as remarked above.

(ii) Assume that $D \subset W_{0} \cup W_{1} \cup W_{2}$. Let $e=(x, n, i) \in D \cap W_{2}$. Now any $e^{\prime}=\left(x^{\prime}, n^{\prime}, i^{\prime}\right) \in D \cap W_{2}$ must fulfill $n=n^{\prime}$ and $i=i^{\prime}$, as upper bounds for other elements of $W_{2}$ lie in $W_{3}$, and by assumption, $D \cap W_{3}=\emptyset$. Accordingly, $D \subset h_{f}$ for any $f \in \mathbb{F}$ for which $f(n)=i$.

(iii) Finally, assume that $D \cap W_{3} \neq \emptyset$, so $D$ contains an element $e=(x, f) \in W_{3}$. As $(x, f)$ and $(y, g)$ with $f \neq g$ do not have an upper bound in $W$, we have $D \cap W_{3} \subset h_{f}$. To establish $D \cap W_{2} \subset h_{f}$, let $e^{\prime}=(y, n, i) \in D \cap W_{2}$. Any upper bound for $e$ and $e^{\prime}$ must be of the form $\left(x^{\prime}, f\right)$, and thus by the definition of the ordering, $e^{\prime}<e$, which means $f(n)=i$, implying $f \in h_{f}$. Thus $D \subseteq h_{f}$.

Next we note that $M_{2}$ has the right topology:

Lemma $5 M_{2}$ fulfills the topological requirements of BST-92, i.e.,

1. Every lower bounded chain $c \subseteq W$ has an infimum (greatest lower bound), inf $c$.

2. If $c \subseteq W$ is an upper bounded chain and $c \subseteq h$ for a history $h$, then there exists a (history-relative) supremum (least upper bound) of $c$ in $h, \sup _{h} c \in h$.

3. W has no minimal or maximal elements. 
Proof (3) is straightforward in virtue of the ordering. For (1) and (2), one only needs to consider the cases in which the supremum or infimum corresponds to $x=0,1$, or 2. In each of these cases, the definition of $W$ secures the right topology. E.g., points $(2, f)$ act both as infima of chains such as $(2+1 / n, f)_{n \in \mathbb{N}}$, and as suprema of chains such as $(2-1 / n, n, i)_{n \in \mathbb{N}}$ in histories $h_{f}$ for which $f(n)=i$.

It remains to prove the prior choice principle.

Lemma $6 M_{2}$ satisfies the prior choice principle: Let $f, f^{\prime} \in \mathbb{F}$, let I be some index set, and let $\left(c_{i}\right)_{i \in I}$ be a lower bounded chain in $h_{f}-h_{f^{\prime}}$. Then there is a lower bound e for $\left(c_{i}\right)_{i \in I}$ that is maximal in $h_{f} \cap h_{f^{\prime}}$.

Proof We first show that any point $e_{n}=(1, n)$ with $f(n) \neq f^{\prime}(n)$ is maximal in $h_{f} \cap h_{f^{\prime}}$. As the $e_{n}$ are elements of any history, they clearly belong to the intersection. For maximality, let $e \in h_{f}$ with $(1, n)<e$. Then either $e=(x, n, i)$ with $f(n)=i \neq f^{\prime}(n)$, whence $e \notin h_{f^{\prime}}$, or $e=(x, f)$, which again is not an element of $h_{f^{\prime}}$.

Concerning the chain $\left(c_{i}\right)_{i \in I}$, we consider two cases. (1) If $(2, f)$ is a lower bound for the chain, then any $(1, n)$ with $n$ s.t. $f(n) \neq f^{\prime}(n)$ will serve as a witness of the prior choice principle. (2) If there are $c_{i}$ for which $c_{i}<(2, f)$, then by the fact that all these elements belong to $h_{f}-h_{f^{\prime}}$ and by linearity, they must all be of the form $(x, n, i)$ for fixed $n$ and $i$-elements of the form $(x)$ or $(x, n)$ belong to $h_{f} \cap h_{f^{\prime}}$, and elements $(x, n, i)$ and $\left(x^{\prime}, n^{\prime}, i^{\prime}\right)$ for $n \neq n^{\prime}$ or $i \neq i^{\prime}$ are incomparable. Thus, $(1, n)$ is a lower bound for the chain $\left(c_{i}\right)_{i \in I}$.

$M_{2}$ is certainly a funny model: It witnesses the strange combinatorics outlined at the beginning of this section; all of the denumerably many points $(1, n), n \in \mathbb{N}$, are binary splitting points, and yet there is no history in which infinitely many " 0 " outcomes occur. As intended, the funniness of $M_{2}$ is not covered by the existing notions of funny business:

Fact $3 \mathrm{M}_{2}$ does not contain a case of primary SLR modal-correlation funny business (nor, in view of Theorem 1, any of the three other cases of definitions 2, 3, or 4).

The proof has already been given above, in motivating the combinatorics used in $M_{2}$.

\section{Combinatorial funny business}

The key idea behind primary SLR modal-correlation funny business (Def. 1) is that there are two well-behaved set-ups (initials $I_{i}$ and outcomes $\Pi_{i}, i=1,2$ ) that one would expect to combine smoothly (since $I_{1}$ SLR $I_{2}$ ), but the histories that would witness smooth combination are missing $\left(\Pi_{1}\left\langle h_{1}\right\rangle \cap \Pi_{2}\left\langle h_{2}\right\rangle=\emptyset\right)$. This is an intrinsically binary concept that, as shown, does not cover some troublesome infinite structures. However, one can generalize by spelling out "well-behaved set-up" in terms of primary transitions (irreducible indeterministic transitions $t_{i}=e_{i} \longmapsto H_{i}$ ), as follows: 
Definition 5 (Combinatorial consistency) A set $T$ of basic transitions is combinatorially consistent iff for any $t_{i}, t_{j} \in T$ :

1. if $e_{i}=e_{j}$, then $H_{i}=H_{j}$ (i.e., $t_{i}=t_{j}$ );

2. if $e_{i}<e_{j}$, then $H_{\left(e_{j}\right)} \subseteq H_{i}$ (i.e., $t_{i}<t_{j}$ );

3. if $e_{j}<e_{i}$, then $H_{\left(e_{i}\right)} \subseteq H_{j}$ (i.e., $t_{j}<t_{i}$ );

4. if $e_{i}$ and $e_{j}$ are incomparable, then $e_{i} \operatorname{SLR} e_{j}$.

As one would expect, sets of transitions that are in fact consistent are also well-behaved according to the definition:

Lemma 7 If $T$ is consistent, then it is also combinatorially consistent.

Proof Assume $T$ is combinatorially inconsistent. Thus, there are $t_{i}, t_{j} \in T$ violating one of the four clauses from the definition. In each of these cases, clearly $H_{i} \cap H_{j}=\emptyset$.

The other direction does not hold in general, but if it fails, something at least mildly counterintuitive is going on: The set $T$ is well-behaved, but the combinatorics do not work out as expected. Thus we define:

Definition 6 (Combinatorial funny business) $T$ constitutes a case of combinatorial funny business iff $T$ is combinatorially consistent, but $H_{T}=\emptyset$.

Fact 4 Both $M_{1}$ and $M_{2}$ are cases of combinatorial funny business.

Proof The initials of indeterministic transitions in both these structures are pairwise space-like related, so the corresponding sets of transitions with outcomes "all 0" fulfill clause (4) of Definition 5-but as noted, there is no history in these structures witnessing the combination of these outcomes.

As we showed at the end of the previous section, the notion of primary SLR modal-correlation funny business does not cover $M_{2}$ (nor, by the light of Theorem 1 , does any of the other existing notions). However, the previous notions smoothly embed into the new one:

Lemma 8 The new notion of combinatorial funny business $(C F B)$ relates to the old notion of primary SLR modal-correlation funny business (PSLRMCFB) in the following ways:

1. CFB is an extension of the notion of PSLRMCFB: Every case of PSLRMCFB is a case of $C F B$.

2. $C F B$ is a proper extension of PSLRMCFB: There are cases of CFB that are not cases of PSLRMCFB.

3. For finite $T, C F B$ is equivalent to PSLRMCFB. ${ }^{4}$

\footnotetext{
${ }^{4}$ However, it is not the case that every case of PSLRMCFB is a case of CFB with finite $T$ - the sets may be infinite in PSLRMCFB too, only the setup is "intrinsically binary".
} 
Proof sketch (1) Given a case of PMCFB $\left(I_{i}, h_{i}, i=1,2\right)$, the two initials $I_{1}$ and $I_{2}$ consist of pairwise SLR initials and are themselves consistent, so

$$
T=\left\{e \longmapsto \Pi_{e}\left\langle h_{1}\right\rangle \mid e \in I_{1}\right\} \cup\left\{e \longmapsto \Pi_{e}\left\langle h_{2}\right\rangle \mid e \in I_{2}\right\}
$$

is combinatorially consistent, but $T$ is inconsistent by assumption. Thus, $T$ is a case of CFB. (2) follows from Facts 3 and 4. For (3), cf. Lemma 2 of Müller (2005).

\section{Explanatory funny business}

Through the new notion of "combinatorial funny business" we have reached our aim of providing for an extended notion of "funny business" that would apply to troublesome infinite cases. Specifically, Lemma 8 shows that the new definition extends the previous "modal correlation" reading of "funny business", PSLRMCFB. By Theorem 1 , the new definition also extends the other three existing notions, some-cause-likelocus-not-in-past funny business (SCLLNIPFB), no-prior-screener-off funny business (NPSOFB), and no-prior-common-cause-like-locus funny business (NPCCLLFB). It would still be nice to see in a more direct way how the new definition extends these three notions, since their motivation is somewhat different from PSLRMCFB. In the latter case, the guiding idea was "wrong kind of combinatorics" (there is a case of inconsistency where one would not expect it). In the former three cases, the guiding idea may be seen to be "warped explanatory account": Each of the three definitions states that there is a case of inconsistency that one cannot make intelligible. SCLLNIPFB states that an explanation (a cause-like locus) is available, but that it does not bear the right kind of spatio-temporal relation to the outcome O. NPSOFB and NPCCLLFB both state that an explanatory account of the right sort cannot be found at all.

By moving from general spatio-temporal/modal structures to sets of basic transitions, we can give a simpler analysis of this kind of "failure of an attempted explanatory account". The guiding idea is the following: If a set of transitions $T$ is inconsistent (the transitions have no joint outcome), then we can make this inconsistency intelligible by showing that $T$ in some way conflates causal alternatives. Alternatives cannot occur together, so if one is presented with a set $T$ in which alternatives are mixed, one readily understands why $T$ should be inconsistent. This may all be well, but what does "causal alternatives" mean? For sets of transitions, there is at least one clear answer, captured by the following definition:

Definition 7 (Blatant inconsistency) A set $T=\left\{e_{i} \longmapsto H_{i} \mid i \in I\right\}$ of transitions is blatantly inconsistent iff there are $t_{i}, t_{j} \in T$ s.t. $e_{i}=e_{j}$, but $H_{i} \neq H_{j}$.

Thus, blatant inconsistency means that in $T$, two local causal alternatives, $t_{i}$ and $t_{j}$, both with the same initial but with different outcomes, are thrown together.

If a given inconsistent set $T$ is blatantly inconsistent, then an account of why it is inconsistent is already available. More generally, one may hope that for inconsistent $T$, one could make the inconsistency intelligible by showing how $T$ could be viewed in such a way as to reveal some blatant inconsistency. In order to get rid of this metaphor, we propose the following notion of "explanation seeking" downward extension: 
Definition 8 (Downward extension) The set $T^{*}$ is a downward extension of $T$ iff (1) $T \subseteq T^{*}$ and (2) for any (new) $t^{*} \in\left(T^{*}-T\right)$, there are (already) $t_{i}, t_{j} \in T$ s.t. (a) $e^{*} \leq e_{i}, e^{*}<e_{j}$ and (b) $t^{*} \not \leq t_{i}, t^{*}<t_{j}$.

That is, in downward extending one may add new transitions $t^{*}=e^{*} \longmapsto H^{*}$ that serve to split off old inconsistencies from one another: the new $t^{*}$ rules out $t_{i}$, but it is compatible with $t_{j}$. These new transitions may help to make the inconsistency of a set $T$ more intelligible by giving more detail.

The definition of "downward extension" is deliberately not meant to single out one ("exhaustive") extension; a given $T$ may have many downward extensions that may be more or less helpful in making $T$ more intelligible. In some cases, however, the extension is unique:

Lemma 9 If $T$ is combinatorially consistent, then $T$ itself is its only downward extension.

Proof Let $T$ be combinatorially consistent, and let $t_{i}, t_{j} \in T$. Let $e^{*}$ fulfill the required clause (2.a) for "downward extension", i.e., $e^{*} \leq e_{i}, e^{*}<e_{j}$. We show that in none of the four cases allowed by Definition 5 can we select $H^{*} \in \Pi_{e^{*}}$ such as to fulfill the second clause (2.b) for "downward extension". (i) $t_{i}=t_{j}$ : (2.b) is contradictory. (ii) $t_{i}<t_{j}$ : if $e^{*}<e_{i}$, then (2.b) fails since $t^{*}<t_{j}$ requires $H^{*}=\Pi_{e^{*}}\left\langle e_{j}\right\rangle$, and by transitivity we get $H^{*}=\Pi_{e^{*}}\left\langle e_{i}\right\rangle$. If, on the other hand, $e^{*}=e_{i}$, then $t^{*}<t_{j}$ requires $t^{*}=t_{i}$. (iii) is symmetrical to case (ii). (iv) $e_{i} \operatorname{SLR} e_{j}$ : with $e^{*}<e_{i}, e^{*}<e_{j}$, there is a history through $e^{*}$ (witnessing the SLR aspect) containing both $e_{i}$ and $e_{j}$, which thus cannot be split at $e^{*}$.

If a given set $T$ is inconsistent, one can hope that it will be possible to arrive at a downward extension of $T$ that is blatantly inconsistent. This would make the inconsistency plainly intelligible. If that hope is frustrated, something funny is going on. Thus we define:

Definition 9 (Explanatory funny business) A set $T$ of transitions is a case of explanatory funny business iff (1) $T$ is inconsistent and (2) there is no downward extension of $T$ that is blatantly inconsistent.

It turns out that the notion of explanatory funny business is equivalent to the previous notion of combinatorial funny business; thus it also applies to troublesome infinite cases.

Theorem 2 In a model of branching space-times, there is a case of explanatory funny business $(E F B)$ iff there is a case of combinatorial funny business (CFB).

Proof " $\Rightarrow$ ": Assume that there is no CFB, and let $T$ be inconsistent. Since there is no CFB, $T$ must be combinatorially inconsistent, i.e., there must be $t_{i}, t_{j} \in T$ violating one of the clauses (1-4) of Definition 5. In each case we can construct a downward extension of $T$ that is blatently inconsistent. 
1. $e_{i}=e_{j}, H_{i} \neq H_{j}: T$ is already blatantly inconsistent.

2. $e_{i}<e_{j}, H_{\left(e_{j}\right)} \nsubseteq H_{i}$ : One may add the transition $e_{i} \longmapsto \Pi_{e_{i}}\left\langle e_{j}\right\rangle$, creating blatant inconsistency.

3. $e_{j}<e_{i}, H_{\left(e_{i}\right)} \nsubseteq H_{j}$ : as in the previous case

4. $e_{i}$ and $e_{j}$ are incompatible (i.e., do not belong to any one history). By the prior choice principle one can find a prior splitting point $e^{*}$ for $e_{i}$ and $e_{j}$ and add $t_{1}^{*}=$ $e^{*} \longmapsto \Pi_{e^{*}}\left\langle e_{i}\right\rangle, t_{2}^{*}=e^{*} \longmapsto \Pi_{e^{*}}\left\langle e_{j}\right\rangle$, creating blatant inconsistency.

Thus, $T$ is not a case of EFB.

“ $\Leftarrow$ ": Let $T$ be a case of CFB, i.e., combinatorially consistent, but inconsistent. By clause (1) of Definition 5, $T$ is not blatantly inconsistent, and by Lemma 9, $T$ is its own unique downward extension. Thus $T$ is a case of EFB.

From Theorems 1 and 2 it is already clear that the notion of EFB is a proper extension of the three previous "explanatory" notions of funny business, SCLLNIPFB, NPSOFB, and NPCCLLFB. Luckily, there is a more perspicuous interdependence: An alternative, direct proof that EFB extends these three notions is readily available. We show the link between EFB and NPCCLLFB explicitly:

Lemma 10 The new notion of explanatory funny business (EFB) relates to the old notion of no prior common cause-like locus funny business (NCCLLFB) in the following ways:

1. EFB is an extension of the notion of NCCLLFB: Every case of NCCLLFB is a case of $E F B$.

2. EFB is a proper extension of NCCLLFB: There are cases of EFB that are not cases of NCCLLFB.

Proof sketch (1) Given a case of NCCLLFB $\left(\mathbf{O}_{i}, h_{i}, i=1,2\right)$, set

$$
T_{i}:=\left\{e \longmapsto \Pi_{e}\langle O\rangle \mid O \in \mathbf{O}_{i}, e=\inf (O)\right\}, \quad i=1,2 ; \quad T:=T_{1} \cup T_{2} .
$$

The $T_{i}$ are consistent, whereas $T$ is inconsistent. If there was a proper extension $T^{*}$ of $T$ that was blatantly inconsistent, there would have to be (new) $t^{*}$ and $t_{1}, t_{2} \in T_{1} \cup T_{2}$ fulfilling the clause for "downward extension" above. As the $T_{i}$ are consistent and thus, combinatorially consistent, Lemma 9 shows that we must have $t_{1} \in T_{1}, t_{2} \in T_{2}$ (modulo relabelling). But then $e^{*}$ would serve to split off $T_{1}$ from $T_{2}$, acting as a common cause, thus violating clause (3) of the definition of NCCLLFB (Def. 4), which requires:

$$
\neg \exists e \exists O_{1} \in \mathbf{O}_{1} \exists O_{2} \in \mathbf{O}_{2}\left(e<O_{1} \wedge e<O_{2} \wedge h_{1} \perp_{e} h_{2}\right)
$$

for histories $h_{i} \in H_{\left\langle\mathbf{O}_{i}\right\rangle}, i=1,2$.

(2) follows from Facts 3 and 4 via Theorem 2. 


\section{Conclusion}

The concept of "modal correlation" seems innocent at first-after all, "modal correlation" just means that some local possibilities do not combine to become global possibilities.

However, formal analysis of the concept of modal correlation reveals some surprising complexities. Getting clear about modal correlations means getting clear about the concept of local and global possibilities first. That in turn presupposes a formal theory of possibility in space and time. Such a theory is provided by BST-92, and preceeding analyses of modal correlations (Belnap 2002, 2003) have accordingly employed that framework.

The present paper has shown that despite the stability of the preceeding analyses, witnessed by the equivalence result of Theorem 1, there are further cases of "funny business" that call for an extension of the previous definitions. We have provided two equivalent, new definitions of "funny business" that spell out what we claim are two main intuitions behind the concept of modal correlations, or "funny business". (1) Local possibilities do not give rise to expected global possibilities. That intuition had found expression in the notion of "primary space-like-related modal-correlation funny business". That notion was based on combining two local possibilities. Our notion of "combinatorial funny business" extends that notion in such a way that infinite sets of local possibilities can be handled smoothly. The extension is natural in that the previous notion appears as a special case (Lemma 8). (2) Global impossibilities do not have the right kind of local explanation. That concept can be made out as a guiding intuition behind the three other proposed definitions of "some cause-like-locus-notin-past funny business", "no-prior-screener-off funny business", and "no-priorcommon-cause-like-locus funny business". Our notion of "explanatory funny business" provides the extension of these concepts that is needed to account for infinite cases. The link is most obvious with respect to "no-prior-common-cause-like-locus funny business", as witnessed by Lemma 10 .

It is our hope that the generality achieved through the present definitions gives, relative to the austere framework of branching space-times, the definite analysis of the notion of "modal correlation".

Acknowledgements Thomas Müller acknowledges support by the Deutsche Forschungsgemeinschaft.

\section{Appendix A}

Some details of branching space-times

BST-92 starts with two primitive notions: "Our world", $W$, whose members are defined as point events, and $\leq$, the "causal order" on $W$. It is assumed that $\leq$ is a dense partial order on $W$ with no maximal elements. A history $h$ is a maximal directed set, where a set is directed if it contains an upper bound for each pair of its members. A set of histories, also called a proposition, is usually denoted by $H$.

$O$ is an outcome chain (nonempty and lower bounded chain, where a set is a chain if each two of its members are comparable by $\leq$ ); provably $O \subseteq h$ for some $h$. It 
is assumed that $O$ has always a unique infimum inf $O$, and it is provable that given $e \in h$, there is an $O$ such that $O \cap h \neq \emptyset$ and $e<O$ and $e=\inf O$. It is also assumed that every upper bounded chain has a supremum in every history to which it belongs. An initial event $I$ is a set of point events all of which are members of some one history, and a scattered outcome event $\mathbf{O}$ is a set of outcome chains all of which overlap some one history. By the prior choice postulate, for every $O \subseteq h_{1}-h_{2}$ there is a point $e \leq O$ that is maximal in the intersection $h_{1} \cap h_{2}$.

$H_{(e)}=\{h \mid e \in h\}$ is the proposition saying that $e$ occurs. More generally, $H_{[I]}=\{h \mid I \subseteq h\}$ is the proposition saying that $I$ occurs. $H_{\langle O\rangle}=\{h \mid h \cap O \neq \emptyset\}$ is the proposition saying that $O$ occurs, and for a scattered outcome event $\mathbf{O}$, the occurrence proposition is $H_{\langle\mathbf{O}\rangle}=\cap_{O \in \mathbf{O}} H_{\langle O\rangle}$. A proposition $H$ is consistent iff $H \neq \emptyset$, and an event of some type $(e, O, \mathbf{O}, I)$ is consistent iff its occurrence proposition is.

Two histories are undivided at $e, h_{1} \equiv_{e} h_{2}$, iff $e$ belongs to their intersection, but is not maximal therein. More generally, $h_{1} \equiv_{I} h_{2}$ iff $h_{1} \equiv_{e} h_{2}$ for every $e \in I$. By the prior choice postulate, undividedness-at- $e$ (and thus, undividedness-at- $I$ ) is an equivalence relation, and the corresponding partition of $H_{(e)}\left(\right.$ of $\left.H_{[I]}\right)$ is denoted $\Pi_{e}$ $\left(\Pi_{I}\right)$. Given $e \in h, \Pi_{e}\langle h\rangle$ is that member of $\Pi_{e}$ to which $h$ belongs; similarly for $\Pi_{I}\langle h\rangle$.

Point events are space-like-related iff they are distinct, not causally ordered and share a history. $I_{1}$ SLR $I_{2}$ means that every point event in $I_{1}$ is space-like related to every point event in $I_{2}$.

$h_{1}$ is separated from $h_{2}$ at $e$, written $h_{1} \perp_{e} h_{2}$, iff $e$ is maximal in $h_{1} \cap h_{2}$. More generally, $h_{1}$ is separated from $H$ at $I$, written $h_{1} \perp_{I} H$, iff for every $h_{2} \in H$ there is some $e \in I$ for which $h_{1} \perp_{e} h_{2}$. Similarly for $H_{1} \perp_{I} H_{2} . h_{1}$ is relevantly separated from $H$ at $I$, written $h_{1} \perp_{I} H$, iff $h_{1}$ is separated from $H$ at $I$, and each $e \in I$ plays a role, i.e., for every $e \in I$ there is some $h_{2} \in H$ s.t. $h_{1} \perp_{e} h_{2}$. I is a cause-like locus for $\mathbf{O}$ w.r.t. $h$ iff $h \perp_{I} H_{\langle\mathbf{O}\rangle}$.

A transition is a pair $\langle I, O\rangle$ of an initial and an outcome, written $I \longmapsto O$. Basic transitions $t_{i}=e_{i} \longmapsto H_{i}$ are from a point $e_{i}$ to one of the elements $H_{i} \in \Pi_{e}$ of the partition of $H_{\left(e_{i}\right)} . T=\left\{t_{i} \mid i \in I\right\}$ is a set of basic transitions, $I$ some index set. We use

$$
H_{T}:=\bigcap_{t_{i} \in T} H_{i}
$$

to stand for the common outcome of $T$. T is consistent iff $H_{T} \neq \emptyset$. On the set of basic transitions, a partial ordering is defined via

$$
t_{i}<t_{j} \quad \text { iff } \quad e_{i}<e_{j} \quad \text { and } \quad H_{\left(e_{j}\right)} \cap H_{i} \neq \emptyset^{5}
$$

We also set

$$
t_{i} \leq t_{j} \quad \text { iff } \quad t_{i}<t_{j} \quad \text { or } \quad t_{i}=t_{j}
$$

\footnotetext{
${ }^{5}$ By transitivity of undividedness this is equivalent to $H_{\left(e_{j}\right)} \subseteq H_{i}$.
} 


\section{References}

Belnap, Nuel (1992). Branching space-time. Synthese, 92, 385-434.

Belnap, Nuel (2002). EPR-like "funny business" in the theory of branching space-times. In T. Placek \& J. Butterfield (Eds.), Non-locality and modality (pp. 293-315). Dordrecht: Kluwer.

Belnap, Nuel (2003). No-common-cause EPR-like funny business in branching space-times. Philosophical Studies, 114, 199-221.

Belnap, Nuel (2005). A theory of causation: causae causantes (originating causes) as inus conditions in branching space-times. British Journal for the Philosophy of Science, 56, 221-253.

Bub, Jeffrey (1997). Interpreting the quantum world. Cambridge: Cambridge University Press.

Einstein, Albert, Podolsky, Boris, \& Rosen, Nathan (1935). Can quantum-mechanical description of physical reality be considered complete? Physical Review, 47, 777-780.

Greenberger, Anton, Horn, Mike, \& Zeilinger, Anton (1989). Going beyond Bell's theorem. In M. Kafatos (Ed.), Bell's theorem, quantum theory, and conceptions of the Universe (pp. 69-74). Dordrecht: Kluwer.

Müller, Thomas (2005). Probability and causation. A branching space-times analysis. British Journal for the Philosophy of Science, 56, 487-520.

Weiner, Matthew, \& Belnap, Nuel (2006). How causal probabilities might fit into our objectively indeterministic world. Synthese, 149, 1-36. 